\title{
ALMOST-CONTINUOUS PATH CONNECTED SPACES
}

\author{
LARRY L. HERRINGTON \\ Department of Mathematics \\ Louisiana State University at Alexandria \\ Alexandria, Louisiana 71402 U.S.A. \\ and \\ PAUL E. LONG \\ Department of Mathematics \\ The University of Arkansas at Fayetteville \\ Fayetteville, Arkansas 72701 U.S.A. \\ (Received October 31, 1980)
}

ABSTRACT. M. K. Singal and Asha Rani Singal have defined an almost-continuous function $\mathrm{f}: \mathrm{X} \rightarrow \mathrm{Y}$ to be one in which for each $\mathrm{x} \in \mathrm{X}$ and each regular-open set $\mathrm{V}$ containing $f(x)$, there exists an open $U$ containing $x$ such that $f(U) \subset V$. A space $Y$ may now be defined to be almost-continuous path connected if for each $y_{0}, y_{1} \in Y$ there exists an almost-continuous $f: I \rightarrow Y$ such that $f(0)=y_{0}$ and $f(1)=y_{1}$. An investigation of these spaces is made culminating in a theorem showing when the almost-continuous path connected components coincide with the usual components of Y.

KEY WORDS AND PHRASES. Almost-continuous functions, path connected spaces. 1980 MATHEMATICS SUBJECT CLASSIFICATION CODES. 54010.

1. INTRODUCTION.

The concept of an almost continuous function $\mathrm{f}: \mathrm{X} \rightarrow \mathrm{Y}$ has been defined in [1] as one in which for each $x \in X$ and each regular-open $V$ containing $f(x)$ there exists an open set $U$ containing $x$ such that $f(U) \subset V$. Using this concept we make the following two definitions:

DEFINITION 1. The function $\mathrm{f}: \mathrm{I} \rightarrow \mathrm{Y}$ is an almost-continuous path (a.c. path) 
from $y_{0}$ to $y_{1}$ if $f$ is almost continuous, $f(0)=y_{0}$ and $f(1)=y_{1}$.

DEFINITION 2. The space $Y$ is a. $\underline{c}$. path connected if for each $y_{0}, y_{1} \in Y$, there exists an a.c. path from $y_{0}$ to $y_{1}$.

The regular-open sets in $\mathrm{Y}$ may be used as a base to form the semi-regular topology $\mathrm{T}_{S}$ on $\mathrm{Y}$ from which $\mathrm{f}: \mathrm{X} \rightarrow(\mathrm{Y}, \mathrm{T})$ is almost-continuous if and only if $\mathrm{f}: \mathrm{X} \rightarrow\left(\mathrm{Y}, \mathrm{T}_{\mathrm{S}}\right)$ is continuous. Thus, Definition 2 may be restated as $(\mathrm{Y}, \mathrm{T})$ is a.c. path connected if for each $y_{0}, y_{1}$ in $Y$, there is a continuous $f: I \rightarrow\left(Y, T_{s}\right)$ such that $f(0)=y_{0}$ and $f(1)=y_{1}$. In view of this observation, many of the known results for path connected spaces in the usual sense also apply to a.c. path connected spaces. For example, if $y_{0} \in Y$, then $Y$ is a.c. path connected if and only if for each $y \in Y$, there is an a.c. path from $y$ to $y_{0}$. Furthermore, slight variations in known results may sometimes be made to easily produce statements concerning a.c. path connected spaces. An example is that if $f: X \rightarrow Y$ is an a1most-continuous surjection and $\mathrm{X}$ is path connected, then $\mathrm{Y}$ is a.c. path connected.

\section{MAIN RESULTS.}

THEOREM 1. Every a.c. path connected space $\mathrm{Y}$ is connected.

PROOF. Assume $Y=U \cup V$ where $U$ and $V$ are open in $(Y, T)$ and $U \Gamma V=\emptyset$. Then $\mathrm{U}$ and $\mathrm{V}$ are regular-open so that $\mathrm{U} L \mathrm{~V}$ is a separation of $\left(\mathrm{Y}, \mathrm{T}_{2}\right)$. But $\left(\mathrm{Y}, \mathrm{T}_{\mathrm{S}}\right)$ is path connected, hence connected. The contradiction implies $\mathrm{Y}$ is connected.

Let $\mathrm{R}$ be the reals with the usual topology, $\mathrm{Q}$ the set of rational numbers and $\mathrm{R}_{\mathrm{Q}}$ the reals with the topology generated by the usual open intervals together with $Q$ as a subbase. Since the semi-regular topology associated with $R_{Q}$ is the usual topology on $R$, it follows that $f: X \rightarrow R_{Q}$ is almost-continuous if and only if $f: X \rightarrow R$ is continuous. Similarly, $f: I \rightarrow R_{Q} \times R_{Q}$ is almost-continuous if and only if $\mathrm{f}: \mathrm{I} \rightarrow \mathrm{R} \times \mathrm{R}$ is continuous. These observations lead to the following example which shows the converse of Theorem 1 is false.

EXAMPLE 1. Let $\mathrm{Y}=\{(\mathrm{x}, \mathrm{y}): \mathrm{y}=\sin (1 / \mathrm{x}), 0<\mathrm{x} \leq 1\} \subset \mathrm{R} \times \mathrm{R}$. Then $\bar{Y}=Y L\{(x, y):-1 \leq y \leq 1\}$ in $R \times R$ as well as $R_{Q} \times R_{Q}$. Hence $\bar{Y}$ is connected, but not a.c. path connected. 
Example 1 also shows that a space may be a.c. path connected but its closure may not be a.c. path connected.

For a given space $Y$, define $x R Y$ to mean there is an a.c. path from $x$ to $y$. We see immediately that $\mathrm{R}$ is an equivalence relation on $\mathrm{Y}$. The resulting equivalence classes are referred to as $\underline{a} . \underline{c}$. path connected components of $Y$. Consideration of the semi-regular topology reveals that each a.c. path connected component in $Y$ is open (and therefore closed) if and only if each point of $Y$ has an a.c. path connected neighborhood.

THEOREM 2. A space $Y$ is a.c. path connected if and only if it is connected and each $y \in Y$ has an a.c. path connected neighborhood.

PROOF. If $\mathrm{Y}$ is an a.c. path connected space, then $\mathrm{Y}$ is connected by Theorem 1 and each $y \in Y$ has an a.c. path connected neighborhood by the remarks preceeding Theorem 2 .

Conversely, the hypothesis and the remarks preceeding Theorem 2 show that the only a.c. path connected component of $\mathrm{Y}$ is $\mathrm{Y}$ itself. Therefore, $\mathrm{Y}$ is a.c. path connected.

THEOREM 3. Let $\mathrm{Y}$ be a space. If (a) each a.c. path connected component in $\mathrm{Y}$ is open or (b) if each point $y \in Y$ has an a.c. path connected neighborhood, then the a.c. path connected components of $\mathrm{Y}$ coincide with the usual components of $\mathrm{Y}$.

PROOF. The remarks preceeding Theorem 2 show that conditions (a) and (b) are equivalent. So if we assume that each a.c. path connected component of $Y$ is open, then each point of $\mathrm{Y}$ has an a.c. path connected neighborhood. In particular, the a.c. path connected component [y] is an a.c. path connected neighborhood of $y$. Thus, [y] is connected by Theorem 1. It follows that [y] $\subset \mathrm{c}(\mathrm{y})$, where $\mathrm{C}(\mathrm{y})$ is the usual component of $\mathrm{y} \in \mathrm{Y}$. Since [y] is both open and closed in $\mathrm{Y},[\mathrm{y}]$ is both open and closed in $\mathrm{C}(\mathrm{y})$. But $\mathrm{C}(\mathrm{y})$ connected implies [y] $=\mathrm{C}(\mathrm{y})$.

\section{REFERENCES}

1. SINGAL, M. K. and SINGAL, Asha Rani Almost-Continuous Mappings, The Yokohama Math. J. 16 (1968) pp. 63-73. 


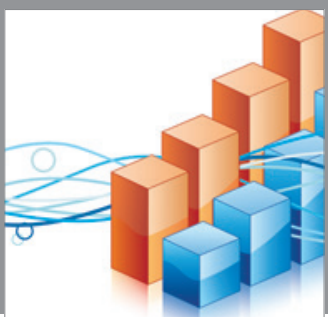

Advances in

Operations Research

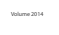

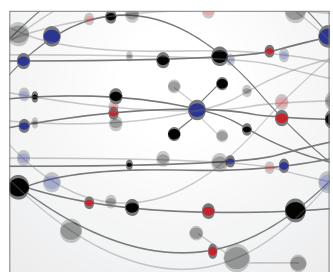

\section{The Scientific} World Journal
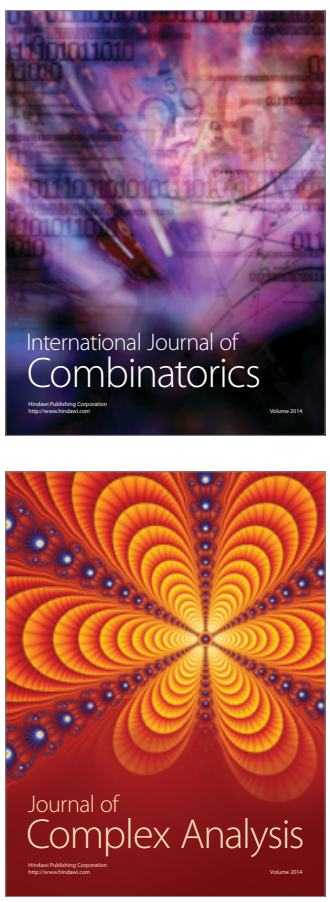

International Journal of

Mathematics and

Mathematical

Sciences
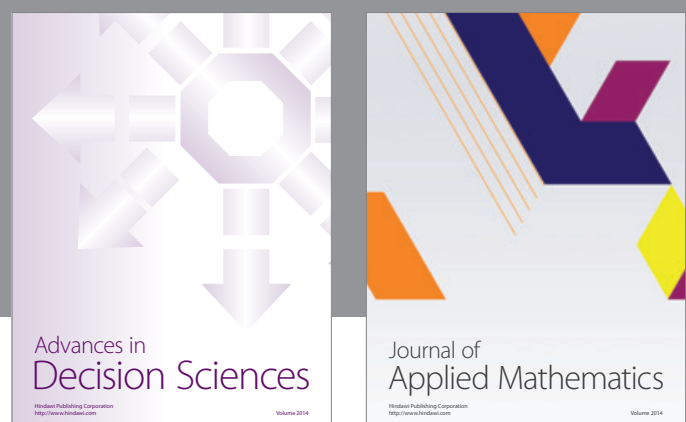

Journal of

Applied Mathematics
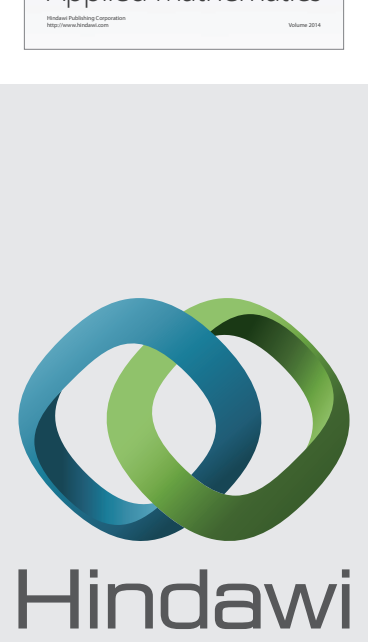

Submit your manuscripts at http://www.hindawi.com
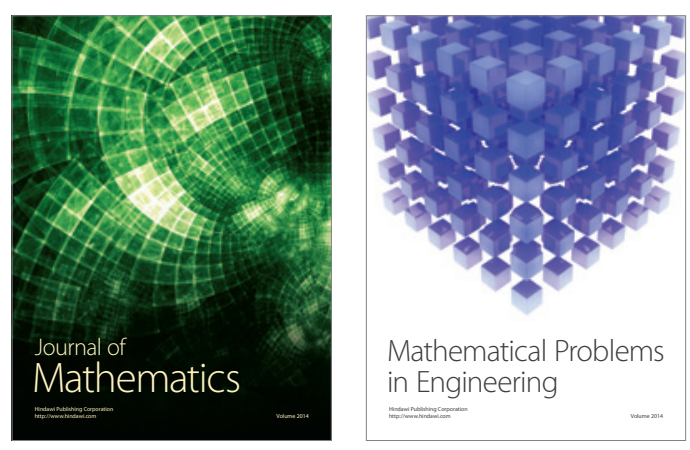

Mathematical Problems in Engineering
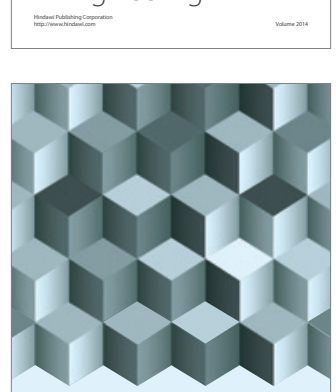

Journal of

Function Spaces
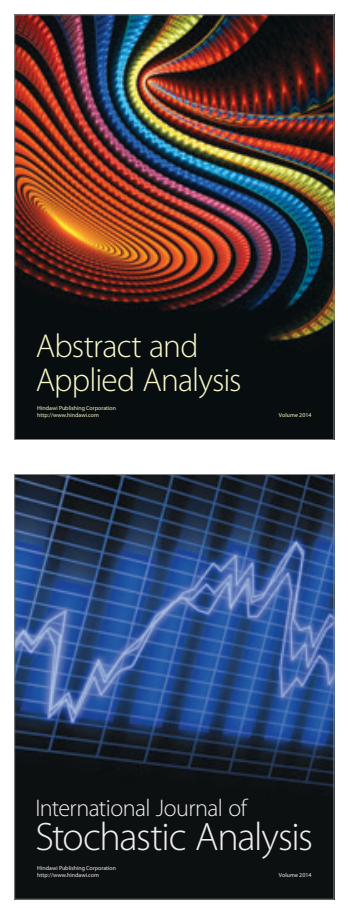

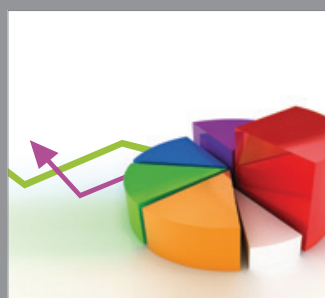

ournal of

Probability and Statistics

Promensencen
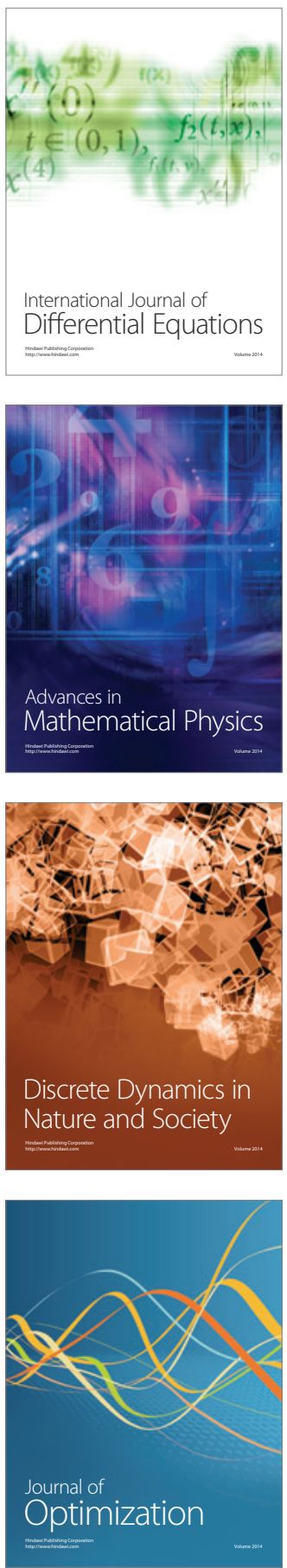